\title{
ARTICLE \\ Nausea in the peri-traumatic period is associated with prospective risk for PTSD symptom development
}

\author{
Vasiliki Michopoulos ${ }^{1,2}$, Jessica Maples-Keller ${ }^{1}$, Elizabeth I. Roger ${ }^{1,3}$, Francesca L. Beaudoin ${ }^{4}$, Jennifer A. Sumner (iD ${ }^{5}$, \\ Barbara O. Rothbaum ${ }^{1}$, Lauren Hudak ${ }^{6}$, Charles F. Gillespie ${ }^{1}$, Ian M. Kronish ${ }^{5}$, Samuel A. McLean ${ }^{7}$ and Kerry J. Ressler ${ }^{1,8}$
}

While nausea often develops following exposure to trauma, little is known regarding the relationship between peri-traumatic nausea and prospective risk for developing posttraumatic stress disorder (PTSD). We examined the association between peritraumatic nausea and PTSD symptom development in three independent cohorts. Participants were recruited from (1) the Emergency Departments (ED) at Grady Memorial Hospital (GMH) in Atlanta, GA, (2) from multiple other ED sites in the TRYUMPH Research Network, and (3) from the ED during evaluation for suspected acute coronary syndrome in the REACH cohort. Administration of IV ondansetron, the most predominant antiemetic used at GMH, was used as a surrogate marker for nausea in the initial GMH cohort; nausea was then directly assessed in the internal validation at GMH, and within the replication TRYUMPH Research Network and REACH cohorts. In the GMH cohort $(N=363)$, ondansetron administration was associated with increased 1and 3-month posttrauma PTSD symptoms in adjusted models (all $p^{\prime} s<0.05$ ). In the GMH internal validation, nausea significantly predicted 1 month $(p=0.009 ; n=68)$ and 3 month $(p=0.029 ; n=54)$ PTSD symptoms. In the TRYUMPH cohort $(N=1846)$, patient reported nausea in the ED was significantly associated with increased PTSD symptoms $(p=0.009)$ in adjusted models. In the REACH cohort $(N=758)$, peri-traumatic nausea was associated with PTSD symptom severity at the 1-month follow-up in adjusted models ( $p$ 's $\leq .008$ ). The current prospective data from three independent cohorts suggest that peri-traumatic nausea is a prospective predictor of PTSD symptom development. Further studies are needed to determine the mechanistic role of nausea as an intermediate phenotype of PTSD risk.

Neuropsychopharmacology (2019) 44:668-673; https://doi.org/10.1038/s41386-018-0276-5

\section{INTRODUCTION}

Posttraumatic stress disorder (PTSD) is a common and severe psychiatric illness characterized by re-experiencing, avoidance and hyperarousal symptoms following trauma [1, 2]. PTSD has significant detrimental effects on quality of life and is related to other adverse health outcomes [3]. Multiple factors are known to influence individual vulnerability to PTSD development, including personal and family history of psychopathology and trauma, initial physiological and emotional responses, and experiences during trauma such as dissociation, and loss of consciousness and traumatic brain injury [4-6]. Additionally, severity and duration of trauma, previous childhood abuse, lack of family and social support and female sex [2] are risk factors for the development of PTSD. While these data suggest that the field has identified some risk factors for PTSD, few studies have described robust prospective risk factors for PTSD development in the aftermath of trauma exposure. Given encouraging results on the effect of immediate psychological and pharmacological interventions in reducing PTSD development $[7,8]$, characterizing additional prospective risk factors could help to better identify individuals who may benefit from intervention in the acute aftermath of trauma.
Exposure to trauma is associated with subsequent persistent somatic symptoms, such as nausea, dizziness, insomnia, fatigue, headache, constipation, or diarrhea $[9,10]$. In a longitudinal study of adolescent females, those who had reported more lifetime violence also experienced more frequent and chronic somatic symptoms, including headaches, stomach aches, and cold sweats [11]. Higher rates of somatic symptoms in female military veterans are associated with violence exposure [12] and PTSD [13]. Interestingly, gastrointestinal (GI) symptoms in particular are highly prevalent in children exposed to violence [14]. A multicenter cross-sectional study reported Gl complaints were prevalent in one-fourth of patients diagnosed with PTSD [15]. In a previous prospective observational investigation of delirium in elderly surgical patients, postoperative nausea, and vomiting was associated with PTSD 3 months following surgery [16]. While PTSD is comorbid with $\mathrm{Gl}$ somatic symptoms following trauma exposure, little is known regarding the relationship between such symptoms in the peri-traumatic period and subsequent PTSD symptom development. For this reason, we chose to prospectively analyze the long-term psychiatric outcomes associated with experiencing nausea, a measure of $\mathrm{Gl}$ distress, as a trauma patient, a prevalent somatic complaint during early trauma care

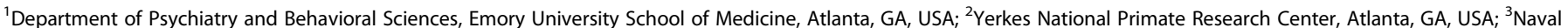

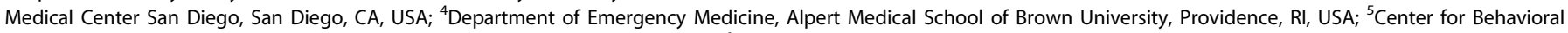

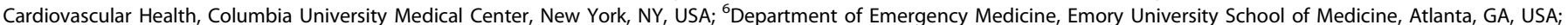

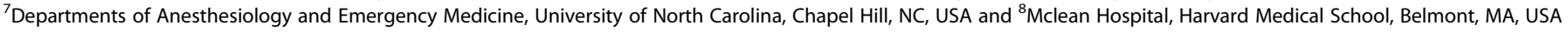
Correspondence: Vasiliki Michopoulos (vmichop@emory.edu)

Received: 9 August 2018 Accepted: 10 November 2018

Published online: 21 November 2018 
across a broad range of trauma mechanisms. To our knowledge, this is the first study to prospectively investigate the association between peri-traumatic nausea and PTSD symptoms.

We examined the relationship between peri-traumatic nausea and future PTSD symptoms in trauma survivors across three samples, including an urban emergency department (ED) trauma sample, a motor vehicle collision (MVC) trauma sample recruited across eight ED sites, and a sample of patients presenting to the ED with suspected acute coronary syndrome (ACS). We hypothesized that the experience of nausea would be predictive of later development of PTSD symptoms, based on previously reported associations of PTSD and somatization [9, 10]. In our initial analysis, ondansetron (Zofran) administration was used as a surrogate marker for the experience of nausea, as it is given regularly in the ED setting as an antiemetic [17]. We also performed subsequent analyses in an internal validation study and two external replication studies in which peri-traumatic nausea symptoms were assessed through patient interview rather than through receipt of antiemetics in the ED.

\section{METHODS}

Grady Memorial Hospital, Atlanta GA

Participants. Participants were recruited from the ED at Grady Memorial Hospital in Atlanta, GA. All study procedures were reviewed and approved by the Emory Institutional Review Board (IRB) and the Grady Hospital Research Oversight Committee. Inclusion criteria included experience of a DSM-IV criterion A trauma in the last $24 \mathrm{~h}$ [18]. Participants were between the ages of 18 and 65 . They were able to provide informed consent, understood and spoke English, and had a phone to allow contact for follow-up appointment scheduling. Exclusion criteria included self-report of current or past history of mania, schizophrenia, other psychoses or prominent suicidal ideation in the last month. Individuals were also excluded upon medical chart review for being intoxicated or not alert, in severe pain, active labor, respiratory distress, anticipating immediate admission or surgery, medically unstable, or hemodynamically compromised in any way.

Procedures. Eligible patients were approached after initial medical evaluation, appropriate laboratory testing, and medical clearance had occurred. Once a patient agreed to participate and signed informed consent, trained assessors collected demographic information and assessments that included information on prior trauma, substance abuse, current and past depression and PTSD symptoms, and details concerning the presenting trauma. Measures included the standardized trauma interview, beck depression inventory to assess baseline depressive symptoms [19], PTSD Diagnostic Scale that captures prior trauma exposure and pre-existing PTSD symptoms [20], and the childhood trauma questionnaire [21]. Participants returned at 1 and 3 months following their ED visit for assessment sessions. The modified PTSD Symptom Scale (mPSS) [22] was used to assess PTSD symptoms at follow-up. Inter-rater reliability was $97 \%$.

Ondansetron administration as a proxy for nausea. Ondansetron administration in the ED was determined by electronic medical records. Assessors at baseline and follow-up visits were unaware of ondansetron administration status. Ondansetron (a single $4 \mathrm{mg}$ IV dose) was administered for reported nausea or prophylactically upon opiate administration to prevent nausea as part of prehospital and ED standard of care procedures by healthcare providers. The assessors for the current observational study were not involved in the medical decision-making or the administration of ondansetron. Less than $1 \%$ of the sample received other antiemetic medications (prochlorperazine, promethazine, and metoclopramide).
Internal validation with self-reported worst nausea. Given the significant differences identified in PTSD symptoms using ondansetron as a proxy for nausea, we then included an item in the baseline ED assessment in which participants were asked, "Please rate the worse nausea level since the event occurred" on a scale from 1 to 10 , with 1 representing no nausea, 5 representing moderate nausea, and 10 representing severe nausea.

Statistical analyses. Two analyses of variance were run to examine differences in PTSD symptoms at 1 month and 3 months posttrauma (primary outcomes) between those receiving versus not receiving IV ondansetron while controlling for opiate administration. For the internal validation cohort, multiple regression analyses were used to assess whether self-reported worst nausea level predicted PTSD total symptom severity at 1and 3 months posttrauma, adjusting for age, trauma severity, baseline PTSD and depression symptoms, and ondansetron and opiate administration. Variables with missing data were excluded from the GMH analyses. The data was analyzed using SPSS (v.20) and alpha level was set at $p \leq 0.05$ for statistical significance.

TRYUMPH Research Network cohort

Participants. Participants were enrolled as part of two large, multicenter prospective cohorts of adult patients (one of European-Americans and one of African-Americans) that recruited from eight EDs as previously described [23]. The study was approved by the IRB at each of the study sites, and all participants provided written informed consent. Patients age 18-65 who presented to the ED within $24 \mathrm{~h}$ after a minor MVC were screened for eligibility. Patients were excluded if they were admitted to the hospital, had fractures other than phalangeal fractures, had more than four lacerations requiring sutures or a single laceration more than $20 \mathrm{~cm}$ in length, or had intracranial or spinal injuries (fracture, dislocation, or new neurologic deficit). Patients with altered mental status, pregnant, unable to speak and read English, and prisoners were excluded, as were patients currently taking betablockers or high dose opioid pain medication on a daily basis.

Procedures. After a patient was deemed eligible, signed informed consent was obtained. Assessments were completed on webbased questionnaires administered by trained research assistants. The interview included demographic information (e.g., age, sex, education level, income level, employment status, and marital status) and information regarding the collision. Peri-traumatic distress in the ED was assessed utilizing the Peri-traumatic Distress Inventory (PDI) [24]. Information extracted from medical record included past medical history, vital signs at presentation, and injury data. Medications administered in the ED, including ondansetron, were extracted from medical records. Less than $1 \%$ of the sample received other antiemetic medications (prochlorperazine, promethazine, and metoclopramide).

ED nausea and follow-up PTSD symptom assessments. Nausea symptom burden was assessed by asking participants to rate the severity of nausea on a zero to ten scale at the ED interview. PTSD symptoms were measured at 6-week follow-up using the Impact Event Scale-Revised (IES-R), wherein items are rated on a 5-point scale ranging from 0 ("not at all") to 4 ("extremely"), yielding a total score (range of $0-88$ ) [25].

Data analyses. Multivariable linear regression was performed to examine the relationship between the receipt of ondansetron and IES-R scores 6-weeks after MVC. The multivariable regression models were adjusted for age, race, sex, income, education, ED peri-traumatic distress, and ED nausea. Descriptive and inferential statistics were performed using STATA 13.0 statistical software (StataCorp. L.P., College TX). Variables with more than $>5 \%$ missing data were handled using multiple imputation $(m=20)$. 

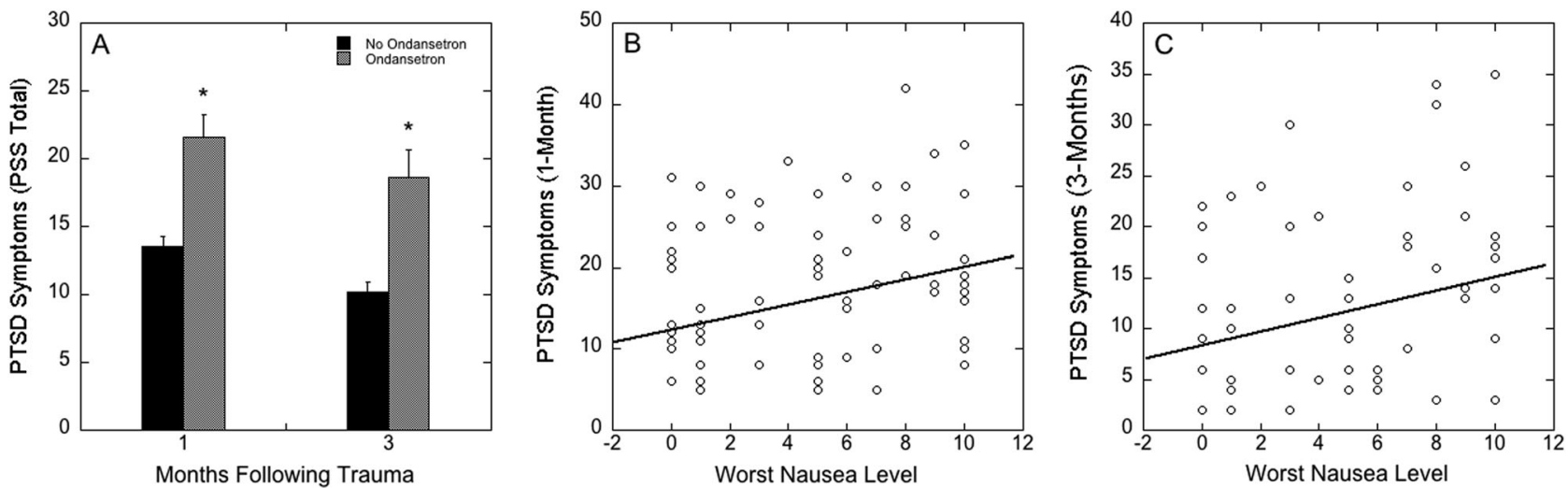

Fig. 1 Ondansetron administration during early trauma care was associated with increased overall PTSD symptoms at both 1- and 3 months posttrauma in the initial GMH sample controlling for ED opiate administration (a; mean \pm SEM). Self-reported worse nausea level in the peritraumatic period is associated with greater PTSD symptoms at both 1 month $(r=0.36 ; p=0.002 ; \mathbf{b})$ and 3 months ( $r=0.39 ; p=0.003$; ) posttrauma

Imputation was performed using logistic regression for binary variables, ordinal logistic regression for ordinal variables, linear regression for continuous variables and predictive mean matching for semi-continuous variables. Over $95 \%$ of the baseline covariates had no more than $1 \%$ missing values, and the maximum proportion of missing for any baseline covariate was $6.4 \%$.

\section{REACH cohort}

Participants. English- and Spanish-speaking adults aged 18 years and older were enrolled during evaluation for suspected ACS in the Columbia University Medical Center ED as part of the REactions to Acute Care and Hospitalization (REACH) study [26]. Eligible patients were identified by a provisional diagnosis of "probable ACS" by the treating ED physician, including non-STelevation myocardial infarction on an electrocardiogram and unstable angina. Exclusion criteria included ST-elevation myocardial infarction on an electrocardiogram, which triggers a rapid emergency protocol and transfer to the cardiac catheterization laboratory such that enrollment in the ED was not possible; inability to follow the protocol (due to dementia or substance abuse); need for immediate psychiatric intervention; and lack of availability for follow-up (e.g., due to terminal noncardiovascular illness). The study was approved by the Columbia University Medical Center Institutional Review Board; all participants provided written informed consent. Among those eligible, $61 \%$ enrolled.

Procedures. At study enrollment in the ED, participants completed measures of demographics (age, sex, and race/ethnicity), the presence of typical ACS symptoms in the past $24 \mathrm{~h}$ including nausea and perceptions of threat during ED evaluation (see ref. [27] for details). Medical records were not available for extraction of medications administered in the ED. During inpatient stay or by phone after discharge (median 3 days after enrollment), participants reported information about acute stress disorder (ASD) symptoms due to evaluation for suspected ACS with the ASD Scale [28]. Medical covariates reflecting mortality risk and degree of comorbidity, namely the Global Registry of Acute Coronary Events [29] and the Charlson Comorbidity Index [30], respectively, were calculated from medical records. Hospital discharge diagnosis (ACS; non-ACS) was determined by medical record review conducted by a research nurse and confirmed by a boardcertified cardiologist. Approximately, 1 month after study enrollment, a phone interview was conducted to assess PTSD symptoms that developed in response to the suspected ACS event and related hospital experience.
ED nausea and follow-up PTSD symptom assessments. During evaluation for suspected ACS in the ED, participants reported if they experienced any of several ACS symptoms in the last $24 \mathrm{~h}$ before coming to the hospital. The presence of nausea (yes/no) was queried as part of this ED assessment. At the 1-month followup assessment over the telephone, PTSD symptoms in the last month that developed in response to the "heart problem, emergency room visit, and hospitalization 1-month ago" were assessed using the PTSD Checklist-Stressor Specific version [31]. PTSD symptom severity was calculated by summing responses to the 17 items (Cronbach's alpha $=0.92$ ).

Data analyses. Regression was performed to investigate the relationship between nausea and PTSD scores at 1-month followup; linear regression was used to examine continuous PTSD symptom severity. We first ran unadjusted models in the overall sample of 810 , followed by multivariable regression models adjusted for age, sex, race/ethnicity, ED threat perceptions total score, ASD symptoms total score, GRACE index, Charlson Comorbidity Index, and confirmed ACS at discharge (yes/no) ( $n$ $=758$ ). Missing data were imputed for total scores if $<50 \%$ of the items were missing. The EM algorithm (as implemented in Proc MI of SAS version 9.4) was used to impute the expected values, conditional on the participant's responses to all answered items, for sporadic missing items of the ASD, threat perception, and PTSD total scores. Analyses were performed using SPSS, version 24.

\section{RESULTS}

Grady Memorial Hospital Cohort

The association between ondansetron and PTSD symptom development. A total of 363 individuals were enrolled at Grady Memorial Hospital (GMH) in the initial cohort, with 249 returning for the 1month and 222 for 3-month follow-up assessments. Of the 363 enrolled, $49.7 \%$ were involved in a MVC, $9.3 \%$ in a sexual assault, $6 \%$ in a nonground level fall, $7.4 \%$ in a nonsexual assault, $6.8 \%$ in a pedestrian vs. car incident, $3.8 \%$ in industrial/home accidents, and $5.2 \%$ in motorcycle crash, $4.4 \%$ gunshot would, $2.2 \%$ stab, $0.8 \%$ burn, and $3 \%$ bicycle vs. car accident. The sample was $46.7 \%$ female and racially and ethnically diverse (20.5\% White, $71.5 \%$ Black, 5.5\% Hispanic, and $2.5 \%$ other race/ethnicity), with a mean age of 34.9 years (standard deviation (SD) $=12.4$, range: $18-64)$. Of the 363 participants enrolled, 66 received IV ondansetron and one received IV promethazine for nausea following trauma exposure and 279 did not. Ondansetron administration during early trauma care was associated with increased overall PTSD symptoms at both 1 month $(F=18.3 ; p<0.001)$ and 3 months posttrauma $(F=$ 
21.4; $p<0.001)$ in the initial cohort where ondansetron was used as a surrogate marker for the experience of nausea (Fig. 1a).

Nausea predicts PTSD symptom severity in internal validation. A total of 111 individuals were enrolled at GMH following the addition of peri-traumatic nausea question, with 71 returning for the 1-month and 55 for 3-month follow-up assessments. Of the 111 enrolled, 45.9\% were involved in a MVC, 3.6\% in a sexual assault, $1.8 \%$ in a nonground level fall, $6.3 \%$ in a nonsexual assault, $14.4 \%$ in a pedestrian vs. car incident, $8.1 \%$ in industrial/home accidents, and $2.7 \%$ in motorcycle crash, $9.0 \%$ gunshot would, $2.7 \%$ stab, and $3.6 \%$ bicycle vs. car accident. The sample was $45.9 \%$ female and racially and ethnically diverse $(18.9 \%$ White, $72.1 \%$ Black, $1.8 \%$ Hispanic, and $7.2 \%$ other race/ethnicity), with a mean age of 37.1 years (SD $=13.7$, range: 18-64). Worst nausea during the peri-traumatic period predicted PTSD symptom severity at both 1 month $(r=0.36 ; p=0.002 ; n=71$; Fig. $1 b)$ and 3 months $(r=0.39 ; p=0.003 ; n=55$; Fig. $1 \mathrm{c})$. Multiple linear regression analyses adjusting age, trauma severity, baseline PTSD and depression symptoms, and ondansetron and opiate administration indicated that worst nausea is predictive of PTSD symptom severity at 1 and 3 months (Table 1). Worse nausea significantly predicting PTSD symptoms at 1 month $(n=68, \beta=$ $0.30, p=0.009$, (95\% Cl: $0.26-1.72)$ ) and 3 months posttrauma ( $n$ $=54, \beta=0.32, p=0.029$, (95\% Cl: $0.10-1.81)$ ), whereas ondansetron administration was not a significant predictor at 1 month $(\beta$ $=0.035, p=0.74)$ or 3 months posttrauma $(\beta=-0.032, p=0.80)$ in this sample.

Replication sample investigating peri-traumatic ondansetron and nausea with PTSD in the TRYUMPH cohort

Replication in an independent multivariate cohort of EuropeanAmericans (EA, $n=948$ ) and African-Americans (AA, $n=931$ ) experiencing MVC included a total of 1879 individuals. In both cohorts, slightly more than $60 \%$ of enrolled study participants were female and the median age in both cohorts was 35 years (range: 18-65). Approximately, $7.7 \%$ of participants received

Table 1. Multiple regression with self-reported worse nausea levels and baseline predictors predicting PTSD symptoms $1(n=68)$ and 3 months $(n=54)$ following trauma the internal validation GMH sample

\begin{tabular}{llllll}
\hline PTSD symptoms & $R$ & $R^{2}$ & SE & $\beta$ & $p$ Value \\
\hline 1-month posttrauma & 0.65 & 0.43 & 8.54 & & \\
Age & & & & 0.19 & 0.075 \\
Trauma severity & & & & 0.091 & 0.38 \\
Baseline PTSD & & & & -0.058 & 0.71 \\
Baseline depression & & & & $0.55^{*}$ & 0.001 \\
Ondansetron & & & & 0.035 & 0.74 \\
Opiates & & & & -0.016 & 0.88 \\
Worst nausea & & & & $0.30^{*}$ & 0.009 \\
3 months posttrauma & 0.54 & 0.29 & 8.63 & & \\
Age & & & & 0.14 & 0.084 \\
Trauma severity & & & & -0.004 & 0.98 \\
Baseline PTSD & & & & 0.24 & 0.90 \\
Baseline depression & & & & 0.31 & 0.11 \\
Ondansetron & & & & -0.032 & 0.80 \\
Opiates & & & & 0.13 & 0.31 \\
Worst nausea & & & & $0.32^{* *}$ & 0.029 \\
\hline${ }^{*} p \leq 0.01$. & & & & \\
$*{ }^{*} p \leq 0.05$. & & & & \\
\hline
\end{tabular}

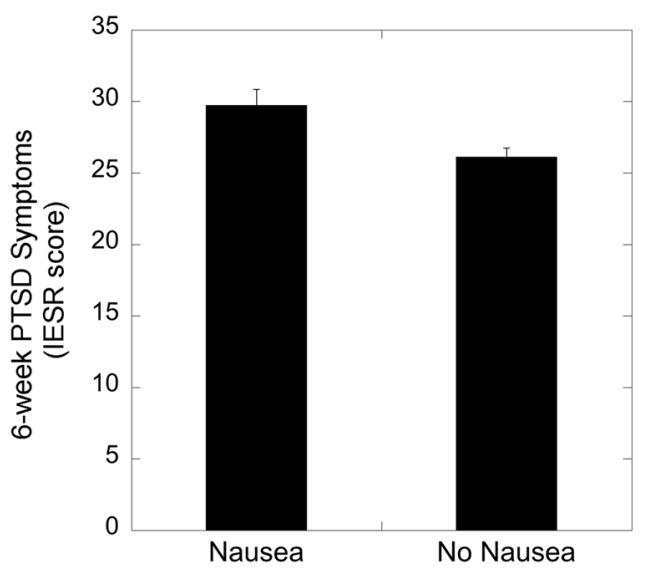

Fig. 2 Mean \pm SEM total posttraumatic stress disorder (PTSD) symptom scores at 6 weeks following an MVC for participants in the TRYUMPH cohort who did and did not report nausea in the emergency department

ondansetron as part of their medical care in the ED (EA: $n=68$, AA: $n=74)$. Ondansetron administration was associated with greater PTSD symptom severity (as measured by IES-R scores) at 6 weeks posttrauma $(\beta=4.3, p=0.032$, (95\% Cl: $0.36-8.3)$ ). However, after adjusting for gender, age, race, income, education, ED peri-traumatic distress, and ED nausea severity, ondansetron administration was not associated with PTSD symptom severity at 6 weeks posttrauma exposure $(\beta=-2.29, p=0.18$ ( $95 \% \mathrm{Cl}:-5.70$ to 1.11)). In contrast, in this adjusted model, ED nausea severity remained significantly associated with increased PTSD symptoms at 6 weeks posttrauma $(\beta=3.00, p=0.009$ (95\% Cl: $0.74-5.27)$ : Fig. 2).

Replication sample investigating nausea with PTSD after suspected ACS in the REACH cohort

A total of 810 participants evaluated for suspected ACS had data on nausea and PTSD symptoms at 1-month follow-up. The sample was $46.8 \%$ female and racially and ethnically diverse $(17.8 \%$ White, 21.0\% Black, 54.7\% Hispanic, and $6.4 \%$ other race/ethnicity), with a mean age of 61.1 years $(S D=13.0$, range: $22-100)$. Nearly, a quarter of the sample reported nausea in the $\operatorname{ED~}(23.6 \%, n=191)$. Nausea was significantly associated with greater PTSD symptom severity at 1-month follow-up in both unadjusted $[\beta=0.13, p<$ $0.0001,(95 \% \mathrm{Cl}: 1.62-5.25), n=810]$ and fully adjusted $[\beta=0.09$, $p=0.006$ (95\% Cl: 0.68-3.96), $n=758$ ] models (Fig. 3).

\section{DISCUSSION}

The current prospective data from three independent observational studies indicate that nausea in the immediate aftermath of trauma is a strong predictor of increased PTSD symptom development prospectively. These findings are most robust at 1 month and 3 months following the index trauma. Initial exploratory analyses indicate an association between ondansetron administration and PTSD symptoms; however, follow-up analyses in an internal validation as well as in the TYRUMPH cohort indicate that when ED nausea severity is included in analyses, ondansetron administration no longer is a significant predictor of PTSD symptoms, whereas peri-traumatic nausea significantly predicts PTSD symptoms prospectively. In an additional replication sample of patients presenting to the ED with suspected ACS and followed for the onset of PTSD symptoms in response to the ACS evaluation and related hospital experience, self-reported nausea also predicted elevated PTSD symptoms at 1-month follow-up (both in terms of continuous symptom severity and screening positive for PTSD). Taken together, these findings suggest that peri- 


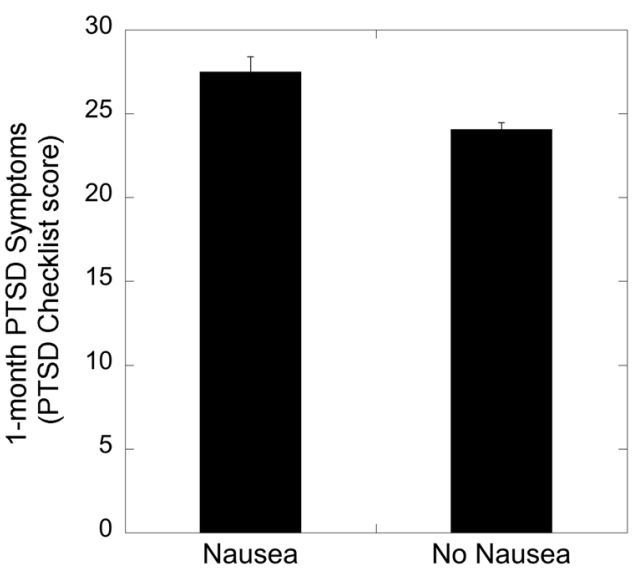

Fig. 3 Mean \pm SEM total posttraumatic stress disorder (PTSD) symptom scores at 1-month follow-up for participants in the REACH cohort who did and did not report nausea in the emergency department. Error bars indicate \pm 1 standard error of the mean

traumatic nausea may identify trauma survivors at risk for the development of PTSD symptoms that may benefit from early interventions that attenuate risk for PTSD [7, 8]. Our results are consistent with multiple published studies that have found associations between somatic symptoms and PTSD following the experience of violence and trauma $[9,10]$. To our knowledge, peritraumatic nausea has not yet been described as a predictor for PTSD. However, postoperative nausea and vomiting has been associated previously with PTSD diagnosis 3 months after surgery in a prospective observational clinical study of elderly patients undergoing general anesthesia [16].

While the mechanism by which nausea may influence PTSD development remains unclear, increased autonomic nervous system activity is common to both conditions. Autonomic nervous system pathways are involved in fear consolidation and associated with aversive learning processes, such as conditioned taste aversion (CTA) [32]. CTA is a classical conditioning process wherein a taste associated with nausea results in learned taste-specific aversion. Appropriate and rapid CTA is an evolutionary advantage; a single taste inducing nausea will stimulate lasting aversion [33]. The actions of calcitonin gene-related peptide (CGRP) in the parabrachial nucleus are necessary for activating the noradrenergic sympathetic system [34] and for a normal CTA response [35]. CGRP itself contributes to fear learning during fear conditioning in rodent models, and antagonism of CGRP impairs the acquisition of contextual fear [36]. While these data suggest that CGRP signaling in the immediate aftermath of trauma may be a future target to investigate for determining the mechanisms by which nausea increases risk for PTSD development, it is important to note that isolating nausea from experiences of threat and fear response activation in the context of trauma exposure is difficult.

In addition to being a physiological read-out of autonomic activation, it is possible that nausea itself contributes to the enhanced consolidation of the trauma memory in those who develop PTSD via other mechanisms. Nausea involves signals from the chemoreceptor zones in the area postrema, the vestibular system, and afferent vagal nerves from the Gl tract sensitive to digested cytotoxic substances [37]. While the central mechanism regulating these signals to generate nausea is still not fully understood [37], nausea may be controlled by $5 \mathrm{HT} 3$ antiemetic agents such as ondansetron [38]. The 5HT3 receptor is expressed centrally in the hippocampus, amygdala, and cortex; all areas that are involved in memory and emotional regulation and are essential for extinction of conditioned fear [39, 40]. Notably, however, our finding that ondansetron dosing in the ED was initially associated with PTSD suggests that suppressing nausea alone, at least within the time frame utilized in these cases, may not be sufficient to decrease PTSD risk.

Peri-traumatic nausea may also symptomatically reflect an acute stress-driven rise in central serotonin [41] following trauma exposure that leads to nausea and which may in parallel also intensify the encoding of trauma-related memories and their later symptomatic expression in the form of PTSD. Indirect support for this hypothesis comes from preclinical and clinical research on the acute effects of selective-serotonin reuptake inhibitors (SSRIs) that are commonly used for treatment of a spectrum of depressive and anxiety disorders. Clinical studies have found repeatedly that SSRIs may intensify anxiety and agitation in a subset of patients during the acute phase of treatment [42] and they may also acutely contribute to nausea and emetogenesis [43]. Although the exact mechanism for the acute clinical effects of SSRIs on anxiety is unknown, one possibility may be that the rapid rise in synaptic serotonin that occurs as a consequence of reuptake inhibition results in anxiogenesis in vulnerable individuals. Translational studies in healthy human subjects have found that acute administration of the SSRI, citalopram, enhances the recognition of fearful faces and increases baseline startle [44]. Similarly, acute administration of escitalopram worsens anxiety and increases plasma cortisol in response to a psychosocial stress task [45]. Collectively, these data suggest that exaggerated serotonergic signaling during acute stress may contribute to both nausea symptoms and intensified encoding of trauma-related memories to elevate risk for PTSD development.

In conclusion, the current study is the first prospective account linking peri-traumatic nausea to significantly increased risk for PTSD. This finding was replicated across three diverse and distinct samples. However, further research is needed to explore the relationship between peri-traumatic nausea and PTSD symptom development, as the current study was not randomized and the possibility remains that other factors may influence the association between nausea and PTSD symptoms development, including ondansetron's direct actions on blocking serotonin 3 receptors. Furthermore, ondansetron was used as a proxy of nausea in the initial Grady study, but nausea did not predict ondansetron in the GMH internal validation, suggesting that there may be other factors associated with ondansetron administration in the ED or peri-traumatic nausea itself that may influence the relationship between the two. Future randomized studies focusing on the association between other peri-traumatic somatic symptoms and subsequent PTSD outcomes are also warranted, as there is a high comorbidity between minor traumatic brain injury and PTSD [5]. Translational research must be undertaken to identify the role of nausea in PTSD development and fear memory consolidation. An exciting possibility is that nausea may represent a targetable intermediate phenotype related to enhanced fear consolidation contributing to PTSD development. Risk factors for PTSD are often difficult to assess in an ED setting, whereas selfreported nausea may be a robust biomarker for PTSD risk that can be efficiently and easily assessed in EDs and may identify individuals who would benefit from early interventions $[7,8]$.

\section{ACKNOWLEDGMENTS}

We would like to particularly thank Alex Rothbaum, Thomas Crow, and Becky Hinrichs for their support and assistance. All of this work would not have been possible without the support of all the nurses, physicians, associate providers, and staff of the Emergency Care Center at Grady Memorial Hospital, the TRYUMPH Research Network sites, and Columbia University Medical Center. Additionally, we would like to acknowledge the patients and families that agreed to participate in these studies.

\section{FUNDING}

This work was supported by the National Institute for Health (R01 MH094757, K.J.R.), the National Institute of Arthritis and Musculoskeletal and Skin Diseases (R01 
AR060852 and R01 AR056328, S.A.M.), the National Institute of Child Health and Human Development (K12 HD085850, V.M.), the National Heart, Lung, and Blood Institute (R01 HL123368, I.M.K.; R01 HL117832; K01 HL130650, J.A.S.) and the Brain and Behavior Research Foundation.

\section{ADDITIONAL INFORMATION}

Competing interests: The authors declare no competing interests.

Publisher's note: Springer Nature remains neutral with regard to jurisdictional claims in published maps and institutional affiliations.

\section{REFERENCES}

1. Gillespie CF, Bradley B, Mercer K, Smith AK, Conneely K, Gapen M, et al. Trauma exposure and stress-related disorders in inner city primary care patients. Gen Hosp Psychiatry. 2009;31:505-14.

2. Kessler RC, Sonnega A, Bromet E, Hughes M, Nelson CB. Posttraumatic stress disorder in the National Comorbidity Survey. Arch Gen Psychiatry. 1995;52:1048-60.

3. Koenen KC, Sumner JA, Gilsanz P, Glymour MM, Ratanatharathorn A, Rimm EB, et al. Post-traumatic stress disorder and cardiometabolic disease: improving causal inference to inform practice. Psychol Med. 2017;47:209-25.

4. Kleim B, Ehlers A, Glucksman E. Early predictors of chronic post-traumatic stress disorder in assault survivors. Psychol Med. 2007;37:1457-67.

5. Blevins CA, Weathers FW, Witte TK. Dissociation and posttraumatic stress disorder: a latent profile analysis. J Trauma Stress. 2014;27:388-96.

6. Roitman P, Gilad M, Ankri YL, Shalev AY. Head injury and loss of consciousness raise the likelihood of developing and maintaining PTSD symptoms. J Trauma Stress. 2013;26:727-34.

7. Holbrook TL, Galarneau MR, Dye JL, Quinn K, Dougherty AL. Morphine use after combat injury in Iraq and post-traumatic stress disorder. N Engl J Med. 2010;362:110.

8. Rothbaum BO, Kearns MC, Reiser E, Davis JS, Kerley KA, Rothbaum AO, et al. Early intervention following trauma may mitigate genetic risk for PTSD in civilians: a pilot prospective emergency department study. J Clin Psychiatry. 2014;75:1380-7.

9. Ulirsch JC, Ballina LE, Soward AC, Rossi C, Hauda W, Holbrook D, et al. Pain and somatic symptoms are sequelae of sexual assault: results of a prospective longitudinal study. Eur J Pain (Lond, Engl). 2014;18:559-66.

10. Gillock KL, Zayfert C, Hegel MT, Ferguson RJ. Posttraumatic stress disorder in primary care: prevalence and relationships with physical symptoms and medical utilization. Gen Hosp Psychiatry. 2005;27:392-9.

11. Halpern CT, Tucker CM, Bengtson A, Kupper LL, McLean SA, Martin SL. Somatic symptoms among US adolescent females: associations with sexual and physical violence exposure. Matern Child Health J. 2013;17:1951-60.

12. Stein MB, Lang AJ, Laffaye C, Satz LE, Lenox RJ, Dresselhaus TR. Relationship of sexual assault history to somatic symptoms and health anxiety in women. Gen Hosp Psychiatry. 2004;26:178-83.

13. Escalona R, Achilles G, Waitzkin $H$, Yager J. PTSD and somatization in women treated at a VA primary care clinic. Psychosomatics. 2004;45:291-6.

14. Bailey BN, Delaney-Black V, Hannigan JH, Ager J, Sokol RJ, Covington CY. Somatic complaints in children and community violence exposure. J Dev Behav Pediatr : JDBP. 2005;26:341.

15. Lowe B, Kroenke K, Spitzer RL, Williams JB, Mussell M, Rose M, et al. Trauma exposure and posttraumatic stress disorder in primary care patients: crosssectional criterion standard study. J Clin Psychiatry. 2011;72:304-12.

16. Drews T, Franck M, Radtke FM, Weiss B, Krampe H, Brockhaus WR, et al. Postoperative delirium is an independent risk factor for posttraumatic stress disorder in the elderly patient: a prospective observational study. Eur J Anaesthesiol. 2015;32:147-51.

17. Milne RJ, Heel RC. Ondansetron. Therapeutic use as an antiemetic. Drugs. 1991;41:574-95.

18. APA (2000). Diagnostic and Statistical Manual of Mental Disorders.

19. Beck AT, Ward $\mathrm{CH}$, Mendelson M, Mock J, Erbaugh J. An inventory for measuring depression. Arch Gen Psychiatry. 1961:4:561-71.

20. Foa EB, Cashman L, Jaycox L, Perry K. The validation of a self- report measure of posttraumatic stress disorder: The Posttraumatic Diagnostic Scale. Psychol Assess. 1997;9:445-51.
21. Bernstein DP, Ahluvalia T, Pogge D, Handelsman L. Validity of the Childhood Trauma Questionnaire in an adolescent psychiatric population. J Am Acad Child Adolesc Psychiatry. 1997;36:340-8.

22. Foa EB, Riggs DS, Dancu CV, Rothbaum BO. Reliability and validity of a brief instrument for assessing post-traumatic stress disorder. J Traum Stress 1993;6:459-73.

23. Linnstaedt SD, Hu J, Liu AY, Soward AC, Bollen KA, Wang HE, et al. Methodology of AA CRASH: a prospective observational study evaluating the incidence and pathogenesis of adverse post-traumatic sequelae in African-Americans experiencing motor vehicle collision. BMJ Open. 2016;6:e012222.

24. Brunet A, Weiss DS, Metzler TJ, Best SR, Neylan TC, Rogers C, et al. The Peritraumatic Distress Inventory: a proposed measure of PTSD criterion A2. Am J Psychiatry. 2001;158:1480-5.

25. Creamer M, Bell R, Failla S. Psychometric properties of the Impact of Event Scale -Revised. Behav Res Ther. 2003:41:1489-96.

26. Sumner JA, Chen $Q$, Roberts AL, Winning A, Rimm EB, Gilsanz $P$, et al. Cross sectional and longitudinal associations of chronic posttraumatic stress disorder with inflammatory and endothelial function markers in women. Biol Psychiatry. 2017;82:875-84.

27. Meli L, Kautz M, Julian J, Edmondson D, Sumner JA. The role of perceived threat during emergency department cardiac evaluation and the age-posttraumatic stress disorder link. J Behav Med. 2018;41:357-63.

28. Bryant RA, Moulds ML, Guthrie RM. Acute Stress Disorder Scale: a self-report measure of acute stress disorder. Psychol Assess. 2000;12:61-68.

29. Eagle KA, Lim MJ, Dabbous OH, Pieper KS, Goldberg RJ, Van de Werf F, et al. A validated prediction model for all forms of acute coronary syndrome: estimating the risk of 6-month postdischarge death in an international registry. J Am Med Assoc. 2004;291:2727-33.

30. Charlson M, Szatrowski TP, Peterson J, Gold J. Validation of a combined comorbidity index. J Clin Epidemiol. 1994;47:1245-51.

31. Weathers F, Litz B, Herman D, Huska J, Keane T (1993). The PTSD Checklist (PCL): reliability, validity, and diagnostic utility.

32. Verbalis JG, McHale CM, Gardiner TW, Stricker EM. Oxytocin and vasopressin secretion in response to stimuli producing learned taste aversions in rats. Behav Neurosci. 1986;100:466-75.

33. Sweatt JD. Chapter 4-Rodent Behavioral Learning and Memory Models. Mechanisms of Memory. Second Edition. London: Academic Press; 2010. p. 76-103.

34. Fisher LA, Kikkawa DO, Rivier JE, Amara SG, Evans RM, Rosenfeld MG, et al. Stimulation of noradrenergic sympathetic outflow by calcitonin gene-related peptide. Nature. 1983;305:534-6.

35. Carter ME, Han S, Palmiter RD. Parabrachial calcitonin gene-related peptide neurons mediate conditioned taste aversion. J Neurosci. 2015;35:4582-6.

36. Sink KS, Davis M, Walker DL. CGRP antagonist infused into the bed nucleus of the stria terminalis impairs the acquisition and expression of context but not discretely cued fear. Learn Mem. 2013;20:730-9.

37. Stockhorst $U$, Enck $P$, Klosterhalfen $S$. Role of classical conditioning in learning gastrointestinal symptoms. World J Gastroenterol. 2007;13:3430-37.

38. Morrow GR, Hickok JT, Rosenthal SN. Progress in reducing nausea and emesis. Comparisons of ondansetron (Zofran), granisetron (Kytril), and tropisetron (Navoban). Cancer. 1995:76:343-57.

39. Jakab RL, Goldman-Rakic PS. Segregation of serotonin 5-HT2A and 5-HT3 receptors in inhibitory circuits of the primate cerebral cortex. J Comp Neurol. 2000;417:337-48

40. Park SM, Williams CL. Contribution of serotonin type 3 receptors in the successful extinction of cued or contextual fear conditioned responses: interactions with GABAergic signaling. Rev Neurosci. 2012;23:555-69.

41. Chaouloff F, Berton O, Mormede P. Serotonin and stress. Neuropsychopharmacology. 1999;21(Suppl. 2):28S-32S.

42. Fergusson D, Doucette $S$, Glass KC, Shapiro S, Healy D, Hebert $P$, et al. Association between suicide attempts and selective serotonin reuptake inhibitors: systematic review of randomised controlled trials. Br Med J. 2005;330:396.

43. McManis PG, Talley NJ. Nausea and vomiting associated with selective serotonin reuptake inhibitors. CNS Drugs. 1997;8:394-91.

44. Browning M, Reid C, Cowen PJ, Goodwin GM, Harmer CJ. A single dose of citalopram increases fear recognition in healthy subjects. J Psychopharmacol. 2007;21:684-90.

45. Garcia-Leal C, Del-Ben CM, Leal FM, Graeff FG, Guimaraes FS. Escitalopram prolonged fear induced by simulated public speaking and released hypothalamic-pituitary-adrenal axis activation. J Psychopharmacol. 2010;24: 683-94. 